\title{
ATN incorporating cerebrospinal fluid neurofilament light chain detects frontotemporal lobar degeneration
}

Katheryn A Q Cousins 1, Jeffrey S Phillips 1, David J Irwin 1, Edward B Lee 2, David A Wolk 2, Leslie M Shaw 2, Henrik Zetterberg 345 6, Kaj Blennow 3 4, Sarah E Burke 1, Nikolas G Kinney 1, Garrett S Gibbons 2, Corey T McMillan 1, John Q Trojanowski 2, Murray Grossman 1

1Department of Neurology, University of Pennsylvania, Philadelphia, Pennsylvania, USA.

2Department of Pathology and Laboratory Medicine, University of Pennsylvania, Philadelphia, Pennsylvania, USA.

3Department of Psychiatry and Neurochemistry, Institute of Neuroscience and Physiology, The Sahlgrenska Academy, University of Gothenburg, Gothenburg, Sweden.

4Clinical Neurochemistry Laboratory, Sahlgrenska University Hospital, Mölndal, Sweden.

5Department of Neurodegenerative Disease, Institute of Neurology, University College London, London, UK.

6UK Dementia Research Institute, University College London, London, UK.

Correspondence should be addressed to Katheryn Cousins or Murray Grossman Department of Neurology

Richards Medical Research Laboratories, Suite 600B 3700 Hamilton Walk

Philadelphia, PA 19104

katheryn.cousins@pennmedicine.upenn.edu ormgrossma@pennmedicine.upenn.edu 
Running head: ATN classifications using NFL in FTLD and AD 


\begin{abstract}
Introduction: The ATN framework provides an in vivo diagnosis of Alzheimer's disease (AD) using cerebrospinal fluid (CSF) biomarkers of pathologic amyloid plaques (A), tangles $(\mathrm{T})$, and neurodegeneration $(\mathrm{N})$. ATN is rarely evaluated in pathologically confirmed patients and its poor sensitivity to suspected non-Alzheimer's pathophysiologies (SNAP), including frontotemporal lobar degeneration (FTLD), leads to misdiagnoses. We compared accuracy of ATN (ATNTAU ) using CSF total tau (t-tau) to a modified strategy (ATNNfL ) using CSF neurofilament light chain (NfL) in an autopsy cohort.
\end{abstract}

Methods: ATNTAU and ATNNfL were trained in an independent sample and validated in autopsy-confirmed $\operatorname{AD}(n=67)$ and FTLD $(n=27)$.

Results: ATNNfL more accurately identified FTLD as SNAP (sensitivity $=0.93$, specificity $=0.94$ ) than ATNTAU (sensitivity $=0.44$, specificity $=0.97$ ), even in cases with co-occurring AD and FTLD. ATNNfL misclassified fewer AD and FTLD as "Normal" (2\%) than ATNTAU (14\%).

Discussion: ATNNfL is a promising diagnostic strategy that may accurately identify both $\mathrm{AD}$ and FTLD, even when pathologies co-occur. 


\section{Introduction}

The 2018 ATN research framework provides a diagnostic algorithm along the different clinical stages of Alzheimer's disease (AD), often termed the AD continuum, using the cerebrospinal fluid (CSF) biomarkers amyloid $\beta$ 1-to-42 peptide $\left(\mathrm{A} \beta_{1-42}\right)$, tau protein phosphorylated at threonine 181 (p-tau), and total tau (t-tau) to determine positive $(+)$ or negative (-) status for amyloid deposition (A), tangle formation (T), and neurodegeneration $(\mathrm{N})^{1}$. Beyond a binary diagnosis of AD or not, ATN aims to provide a more detailed profile: normal status, early Alzheimer's pathologic change, AD, suspected non-Alzheimer's pathophysiology (SNAP), or even concomitant SNAP and AD. While A+ status is excellent at identifying AD A $\beta$ deposition, CSF-based ATN designations may be relatively poor at detecting SNAP, including frontotemporal lobar degeneration (FTLD) ${ }^{2}$. This is due, in part, to the limitations of CSF t-tau as a marker of $\mathrm{N}$ status. Much research evaluating ttau has debated its ability to stage disease severity in $\mathrm{AD}$ or predict conversion to mild cognitive impairment or $\mathrm{AD}^{3-8}$. However, CSF-based ATN designations may be confounded because t-tau concentrations are highly correlated with $\mathrm{p}$-tau ${ }^{9}$; consequently $\mathrm{T}$ and $\mathrm{N}$ status are not orthogonal and there may be little added value to $\mathrm{N}$-status using t-tau ${ }^{10}$.

Beyond its role in staging AD, N status is critical to identifying individuals with SNAP, including concomitant SNAP and AD. This discrimination is important for diagnostic decisions such as determining eligibility for disease-modifying treatment trials, especially in phenotypically similar patients with AD compared to SNAP. However, because CSF ttau is relatively reduced in FTLD ${ }^{13,14}$, CSF-based ATN profiles do not well distinguish patients with FTLD from healthy controls ${ }^{2}$. Commonly co-occurring pathologies in AD and FTLD, demonstrated in autopsy series $^{11}$, can also make stratification of a 
heterogeneous cohort challenging. Indeed, a patient with primary FTLD and secondary AD is likely to have low CSF $A \beta_{1-42}$ levels and be mislabeled as $\mathrm{AD}$, thereby overlooking the primary pathology ${ }^{12}$.

In this study, we compare diagnostic accuracy of CSF t-tau to two alternative markers of N-status: CSF neurofilament light chain (NFL) and the ratio of ventricular-to-brain volume (VbV). NFL is an axonal protein showing elevated CSF levels and promise as a clinically helpful diagnostic marker in several neurodegenerative conditions. The combination of CSF NFL and t-tau was found to have high discriminatory power between AD (higher ttau than FTLD) and FTLD (higher NFL than AD) (REF), a finding validated in several studies, ${ }^{15,16}$.and increased levels of NFL have been related to more severe cognitive impairment and shorter survival in both $\mathrm{AD}$ and $\mathrm{FTLD}^{17-20}$. We also test $\mathrm{VbV}$ as a global neuroimaging metric of $\mathrm{N}$ that is not specific to AD. Evidence shows that enlarged ventricular volume and decreasing total brain volume are associated with various disease features in $\mathrm{AD}^{21-23}$ and $\mathrm{FTLD}^{24,25}$, including progressive cognitive impairment, CSF biofluid changes, genetic status, and degeneration.

We test three alternative ATN frameworks in an autopsy cohort of AD, FTLD, and mixed pathologies, combining $A \beta_{1-42}$ and p-tau with t-tau (ATN $N_{T T}$ ), NFL (ATNFL), or VbV $\left(\mathrm{ATN}_{\mathrm{Vv}}\right)$. Logistic regression trained classification models in independent living cohorts for each marker of $\mathrm{N}$. These models are then applied to our autopsy cohort to determine $\mathrm{N}$ status and test classification accuracy of $\mathrm{ATN}_{\mathrm{TT}}, \mathrm{ATN}_{\mathrm{FL}}$, and ATN $\mathrm{Vv}$. A successful ATN framework will not only detect and stage AD, but should also be sensitive to SNAP with and without AD co-pathology. 


\section{Methods}

\section{Autopsy Subjects}

Inclusion criteria were a primary pathological diagnosis of $\mathrm{AD}(\mathrm{n}=91)$ or FTLD $(\mathrm{n}=62)$ and completed assays for CSF $A \beta_{1-42}$ and p-tau; subsets of these patients had available CSF NFL and CSF t-tau data (69 AD; 27 FTLD) and/or magnetic resonance imaging (MRI) data (13 AD; 35 FTLD). MRI data were collected on the same day as CSF. Subjects were autopsied at the University of Pennsylvania Center for Neurodegenerative Disease Research and and all their data were entered into the Integrated Neurodegenerative Disease Database $^{26,27}$ for subsequent data mining and interrogation. Exclusion criteria included cooccurring neurologic conditions (e.g. stroke, hydrocephalus, chronic traumatic encephalopathy, vascular disease at autopsy), and primary psychiatric disorders (e.g. depression, anxiety). Participants' consent for antemortem data was obtained according to the Declaration of Helsinki and approved by the University of Pennsylvania's Institutional Review Board. This is a retrospective study, and subsets of these data have appeared in previous publications ${ }^{28}(\mathrm{REF})$.

Primary pathological diagnosis was based on the judgment of experienced neuropathologists (JQT, EBL). FTLD pathology was determined by accumulations of misfolded 3R or 4R tau (FTLD-Tau) or transactive response DNA-binding protein of 43 kDa (TDP-43) (FTLD-TDP) ${ }^{29,30}$. Severity of AD neuropathologic change (ADNPC: rated as none or scant/low/intermediate/high) was rated using "ABC" scoring methods ${ }^{31}$. For FTLD-tau cases, mAbs GT-7 and GT-38 immunohistochemistry determined AD Braak Stage since these mAbs are wholly specific for AD tau pathology ${ }^{32,33}$. In addition to ADNPC and FTLD pathology, patients were assessed for the co-occurrence of other pathologic conditions. For AD patients with CSF, 37 of 69 had mixed pathology, which 
included 3 cases with secondary diagnosis of FTLD-tau. For AD patients with MRI, 7 of 13 had mixed pathology, none with co-occurring FTLD. Other co-pathological conditions included hippocampal sclerosis, limbic-predominant age-related TDP-43 encephalopathy (LATE), and $\alpha$-synuclein-positive Lewy bodies ${ }^{29,34}$. For FTLD patients with CSF, 5 of 27 had mixed pathology, 2 with secondary AD (1 High ADNPC and 1 Intermediate ADNPC); the remaining had negligible AD pathologic severity (Low [ $\mathrm{n}=13]$ or were completely negative for tau pathology [n=12]). For FTLD patients with MRI, 4 of 35 had mixed pathology, 1 with secondary AD (1 High ADNPC and 0 Intermediate ADNPC); the remaining had negligible AD pathologic severity (Low [n=17] or none [ $\mathrm{n}=17]$ ). Other copathological conditions included $\alpha$-synuclein-positive Lewy bodies, co-occurring FTLDtau and FTLD-TDP, and hippocampal sclerosis. If no form of clinically meaningful copathology was detected, patients were classified as having "negligible" co-pathology. AD patients with amygdala-predominant Lewy bodies or cerebral amyloid angiopathy were considered to have "negligible" co-pathology.

Table 1 provides descriptive statistics and comparisons for the demographic characteristics for AD and FTLD patients in the autopsy cohort, including age of disease onset (earliest reported symptom), age at CSF/MRI, disease duration (years from onset to CSF/MRI), interval to death (years from CSF/MRI to death), Mini-Mental State Examination $(\mathrm{MMSE})^{35}$, survival (years from onset to death), and years of education. Comparing autopsy patients with CSF (Table 1A) and MRI data (Table 1B), AD patients with MRI data were more impaired on MMSE ( $\mathrm{W}=654, p=0.00056$ ) and significantly younger for age-related variables, including age $(\mathrm{W}=641.5, p=0.014)$, age of onset $(\mathrm{W}=653, p=0.0067)$, interval to death ( $\mathrm{W}=634, p=0.018)$, and age at death $(\mathrm{W}=700, p=0.0014)$. There was no 
difference between AD CSF and MRI cohorts for disease duration, education, CSF A $\beta_{1-42}$ or CSF p-tau (all $p>0.1$ ). For FTLD patients, there were no demographic differences between CSF and MRI cohorts, or between CSF A $\beta_{1-42}$ or CSF $p$-tau levels (all $p>0.3$ ).

Table 1: Demographic Characteristics of Autopsy AD and FTLD Cohort. Descriptive statistics across AD and FTLD with CSF (A) and MRI (B) data. Median and interquartile range (median $[\mathrm{IQR}]$ ) are provided. Mann-Whitney-Wilcox or chi-square tests performed pairwise comparisons; $p$-value is based on pairwise comparison for each variable.

\begin{tabular}{|c|c|c|c|}
\hline A. CSF Autopsy Cohort & $\mathrm{AD}$ & FTLD & $p$ \\
\hline $\mathrm{n}$ & 69 & 27 & \\
\hline Age at CSF (years) & $73.00[67.00,78.00]$ & $63.00[58.50,69.50]$ & $<0.001$ \\
\hline CSF to death (years) & $7.00[5.00,9.00]$ & $4.00[2.50,5.00]$ & $<0.001$ \\
\hline Onset age (years) & $70.00[62.00,76.00]$ & $61.00[55.00,66.00]$ & $<0.001$ \\
\hline Disease Duration (years) & $3.00[2.00,5.00]$ & $3.00[2.00,4.00]$ & 0.533 \\
\hline Education (years) & $16.00[12.00,18.00]$ & $16.00[15.00,17.75]$ & 0.740 \\
\hline $\operatorname{MMSE}(\max =30)$ & $25.00[19.50,27.00]$ & $26.00[25.00,28.00]$ & 0.048 \\
\hline $\operatorname{CSF} A \beta_{1-42}(\mathrm{pg} / \mathrm{mL})$ & $130.18[112.00,160.06]$ & $255.00[221.00,290.50]$ & $<0.001$ \\
\hline CSF p-tau (pg/mL) & $33.00[22.59,63.50]$ & $12.00[8.50,16.50]$ & $<0.001$ \\
\hline Sex $=$ Male $(\%)$ & $36(52.2)$ & $13(48.1)$ & 0.898 \\
\hline Co-pathology $=$ Mixed $(\%)$ & $37(53.6)$ & $5(18.5)$ & 0.004 \\
\hline B. MRI Autopsy Cohort & $\mathrm{AD}$ & FTLD & $\mathrm{p}$ \\
\hline $\mathrm{n}$ & 13 & 35 & \\
\hline Age at MRI (years) & $63.00[60.00,74.00]$ & $62.00[57.50,68.00]$ & 0.443 \\
\hline MRI to death (years) & $4.00[3.00,7.00]$ & $3.00[2.00,5.00]$ & 0.134 \\
\hline Onset age (years) & $60.00[52.00,66.00]$ & $58.00[54.00,65.00]$ & 0.486 \\
\hline Disease Duration (years) & $6.00[6.00,6.00]$ & $2.00[2.00,2.00]$ & 1.000 \\
\hline Education (years) & $16.00[14.00,18.00]$ & $16.00[16.00,18.00]$ & 0.351 \\
\hline $\operatorname{MMSE}(\max =30)$ & $14.50[8.25,19.50]$ & $26.00[20.25,27.75]$ & 0.002 \\
\hline CSF A $\beta 1-42(\mathrm{pg} / \mathrm{mL})$ & $145.00[81.00,160.00]$ & $271.00[224.00,318.00]$ & $<0.001$ \\
\hline CSF p-tau (pg/mL) & $31.00[22.00,38.00]$ & $12.00[9.00,16.50]$ & $<0.001$ \\
\hline Sex $=$ Male $(\%)$ & $9(69.2)$ & $20(57.1)$ & 0.668 \\
\hline Co-pathology $=$ Mixed $(\%)$ & $7(53.8)$ & $4(11.4)$ & 0.007 \\
\hline
\end{tabular}

\section{Independent Sample Participants}

To train logistic regression models, we used independent samples of healthy controls without cognitive impairment (MMSE $\geq 29$ and/or $\mathrm{CDR}=0$ ) and symptomatic patients 
with a familial form of fFTLD ${ }^{36}$ with either CSF NFL and CSF t-tau data (Controls $n=46$; fFTLD n=18 [10 C9orf72; 2 GRN; 3 MAPT; 3 TARDBP]) or with MRI data (Controls $\mathrm{n}=47$; fFTLD n=34 [19 C9orf72; 5 GRN; 8 MAPT; 2 TARDBP]). Participants' consent was obtained according to the Declaration of Helsinki and approved by the University of Pennsylvania's Institutional Review Board. Table 2 provides descriptive statistics for the CSF and MRI independent samples cohorts, including age at CSF/MRI, MMSE, sum of boxes for the modified Clinical Dementia Rating (CDR) scale ${ }^{37}$, and years of education. There were no differences between CSF and MRI cohorts for controls or for fFTLD (all $p>0.1)$.

Table 2: Demographic Characteristics of Independent Training Cohort. Descriptive statistics across controls without cognitive impairment and syndromic fFTLD patients with CSF (A) and MRI (B) data. Median and interquartile range (median [IQR]) are provided. Mann-Whitney-Wilcox or chi-square tests performed pairwise comparisons; $p$-value is based on pairwise comparison for each variable.

\begin{tabular}{rrrr}
\hline A. CSF Training Cohort & Control & fFTLD & $p$ \\
\hline $\mathrm{n}$ & 46 & 18 & \\
Age at CSF (years) & $67.00[61.00,73.75]$ & $60.50[55.25,66.75]$ & 0.004 \\
Education (years) & $16.00[16.00,18.00]$ & $16.00[12.00,19.00]$ & 0.646 \\
MMSE (max =30) & $30.00[29.00,30.00]$ & $27.00[23.50,29.00]$ & $<0.001$ \\
CDR Sum (max =24) & $0.00[0.00,0.00]$ & $5.50[4.00,6.00]$ & $<0.001$ \\
CSF A $\beta$ (pg/mL) & $261.07[232.44,315.25]$ & $266.50[206.75,303.75]$ & 0.586 \\
CSF p-tau (pg/mL) & $21.80[15.00,27.84]$ & $12.50[11.00,15.00]$ & 0.006 \\
Sex = Male (\%) & $12(26.1)$ & $12(66.7)$ & 0.006 \\
\hline B. MRI Training Cohort & Control & fFTLD & $p$ \\
\hline n & 47 & 34 & \\
Age at MRI (years) & $66.00[60.00,72.50]$ & $62.00[54.50,66.00]$ & 0.013 \\
Education (years) & $16.00[14.00,18.00]$ & $16.00[14.25,18.00]$ & 0.368 \\
MMSE (max =30) & $30.00[29.00,30.00]$ & $26.00[20.00,29.00]$ & $<0.001$ \\
CDR Sum (max = 24) & $0.00[0.00,0.00]$ & $6.00[4.00,7.50]$ & $<0.001$ \\
Sex = Male (\%) & $17(36.2)$ & $24(70.6)$ & 0.005 \\
\hline
\end{tabular}




\section{CSF analysis}

CSF samples were collected and measured for NFL using the enzyme-linked immunosorbent assay (ELISA) of UmanDiagnostics (UmanDiagnostics, Umeå, Sweden), and for $A \beta_{1-42}, p$-tau, and t-tau using the xMAP Luminex platform ${ }^{38}$ (Fujirebio, Ghent, Belgium). Laboratory technicians performed CSF analyses blinded to clinical data. Previously established cut-points for CSF A $\beta_{1-42}$ and p-tau determine A-status and T-status in patients ${ }^{39}$.

\section{Imaging analysis}

For both autopsy and independent MRI cohorts, high resolution T1-weighted MRI scans were acquired using the 3 following protocols:

1.) 3.0 Tesla SIEMENS TIM Trio scanner, 8-channel head coil, axial plane with repetition time $=1620 \mathrm{~ms}$, echo time $=3.87 \mathrm{~ms}$, slice thickness $=1.0 \mathrm{~mm}$, flip angle $=$ $15^{\circ}$, matrix $=192 \times 256$, in-plane resolution $0.9766 \times 0.9766 \mathrm{~mm}(\mathrm{n}=62$ independent cohort, $\mathrm{n}=46$ autopsy)

2.) 3.0 Tesla SIEMENS Prisma scanner, 64-channel head coil, sagittal plane with repetition time $2400 \mathrm{~ms}$, echo time $=1.96 \mathrm{~ms}$, flip angle $=8^{\circ}$, matrix $=320 \times 320$, slice thickness $=$ $0.8 \mathrm{~mm}$, in-plane resolution $=0.8 \times 0.8 \mathrm{~mm}(\mathrm{n}=9$ independent cohort, $\mathrm{n}=3$ autopsy $)$

3.) 3.0 Tesla SIEMENS TIM Trio scanner, 64-channel head coil, sagittal plane with repetition time $=2300 \mathrm{~ms}$, echo time $=2.95 \mathrm{~ms}$, slice thickness $=1.2 \mathrm{~mm}$, flip angle $=9^{\circ}$, matrix $=256 \times 240$, in-plane resolution $=1.05 \times 1.05 \mathrm{~mm}(\mathrm{n}=10$ independent cohort $)$ Images were processed and segmented with antsCorticalThickness, built on state-of-theart Advanced Normalization Tools (ANTs; https://github.com/ANTs $X / A N T s)^{40}$. For both 
independent and autopsy MRI cohorts, two experienced raters (SB and NK) evaluated quality of images with scans eliminated for artifacts such as excessive movement or poor segmentation. A subset of autopsy patients had imaging data available (13 AD; 35 FTLD). Segmentation extracted ventricular volume and total brain volume for each subject. We calculated $\mathrm{VbV}$ as the ratio of ventricular to total brain volume, with higher values indicating more severe degeneration.

\section{Training Models}

While established cut-points exist for CSF t-tau ${ }^{39}$, the variability in measurement techniques across different sites for NFL and VbV limits establishment of broadly applicable cut-points. So as not to bias results, we used logistic regression to train classification models in independent living cohorts for all three markers of $\mathrm{N}$. Three logistic regression models (Equations 1, 2, 3) were generated for each marker, which included probability of N-positivity (Control $=0, f F T L D=1)$ as the dependent variable and $\mathrm{t}$-tau, NFL, or $\mathrm{VbV}$ as the independent predictor; age at CSF/MRI and sex were included as covariates. In addition, the VbV model included scanner and pulse sequence as covariates. Because $\mathrm{N}$ metrics were not normally distributed, Tukey's ladder of powers determined transformations for each: $\log (\mathrm{t}-\mathrm{tau}),-\mathrm{NFL}^{-1 / 2}$ and $\mathrm{VbV}^{-1 / 2}$.

[1] $\mathrm{P}(\mathrm{N}+) \sim \log (\mathrm{t}-\mathrm{tau})+$ Age at $\mathrm{CSF}+\mathrm{Sex}$

[2] $\mathrm{P}(\mathrm{N}+) \sim-\mathrm{NFL}^{-1 / 2}+$ Age at $\mathrm{CSF}+\mathrm{Sex}$

$[3] \mathrm{P}(\mathrm{N}+) \sim \mathrm{VbV}^{-1 / 2}+$ Age at CSF + Sex + Scanner + Pulse Sequence

Training models were then used to predict the probability of N-positivity in the test autopsy sample, with $\mathrm{P}(\mathrm{N}+)>0.5$ considered positive for $\mathrm{N}_{\mathrm{TT}}, \mathrm{N}_{\mathrm{FL}}$, and $\mathrm{N}_{\mathrm{Vv}}$. In addition, Analyses 
of covariance (ANCOVAs) performed between-group comparisons and receiver operating characteristic (ROC) curve and area under the curve (AUC) analyses assessed discrimination of patients from controls for each marker. Sensitivity and specificity for each marker is reported based on optimal cutoffs calculated by Youden's index.

\section{Statistical analysis in autopsy cohort}

We compared classification accuracy for each framework $\left(\mathrm{ATN}_{\mathrm{TT}}, \mathrm{ATN}_{\mathrm{FL}}, \mathrm{ATN} \mathrm{VV}_{\mathrm{VV}}\right)$ in patients with negligible co-pathology (AD, FTLD) and mixed pathology ( $\mathrm{AD}_{\text {mixed, }}$ FTLD $_{\text {mixed) }}$. Patients with CSF $A \beta_{1-42} \leq 192 \mathrm{pg} / \mathrm{mL}$ were considered $\mathrm{A}+$ and those with CSF p-tau $\geq 23 \mathrm{pg} / \mathrm{mL}$ were considered $\mathrm{T}+{ }^{39}$. Logistic regression models from independent samples determined $\mathrm{N}_{\mathrm{TT}}, \mathrm{N}_{\mathrm{FL}}$, and $\mathrm{N}_{\mathrm{VV}}$ status for each autopsy case.

Group-wise comparisons evaluated differences across AD, FTLD-TDP, and FTLD-tau patients. ANCOVAs compared each N-marker (t-tau, NFL, VbV) across pathology subtype (AD, FTLD-TDP, FTLD-tau). Genetic status was not known for all autopsy participants and was not included as a factor. When performing comparisons for $\mathrm{VbV}$, MRI specific covariates of scanner and pulse sequence were included in models. Because of unbalanced design, type II sum of squares was calculated. Covariates included copathology status (negligible, mixed), age at CSF/MRI, interval from CSF/MRI to death, sex, and MMSE $(\alpha=0.05)$. MMSE scores were unavailable for 4 CSF participants and 3 MRI participants; education data were unavailable for 2 CSF participants and 1 MRI participant. These missing data were imputed based on the mean of each patient's respective pathology and co-pathology group. As above, transformations were applied to $\mathrm{N}$ metrics, which were not normally distributed: $\log (\mathrm{t}-\mathrm{tau}),-\mathrm{NFL}^{-1 / 2}$ and $\mathrm{VbV}^{-1 / 2}$. 
Finally, ROC curve analyses assessed diagnostic accuracy and AUC of each marker when discriminating AD from FTLD pathology. Sensitivity and specificity for each marker is reported based on optimal cutoffs calculated by Youden's index.

All statistical analyses were conducted in the $\mathrm{R}$ statistical environment ${ }^{41}$, using the Companion to Applied Regression (car ${ }^{42}$, multcomp ${ }^{43}$, and partial ROC (pROC) $)^{44}$ packages.

\section{Results}

Training models of $N$ in independent living cohorts of controls and fFTLD

Demographic characteristics are detailed in Table 2A for subjects with CSF data, and in Table 2B for subjects with MRI data. Cognitively unimpaired controls were significantly older than fFTLD patients with CSF data (W=607.5, $p=0.0039)$ and fFTLD patients with MRI data (W=1058, $p=0.013$ ); controls also had a higher proportion of females than fFTLD patients with CSF data $\left(\chi^{2}(1)=7.4, p=0.0064\right)$ and fFTLD patients with MRI data $\left(\chi^{2}(1)=8, p=0.0046\right)$. There was no difference between groups for years of education obtained.

Between-group comparisons of N metrics showed that fFTLD had significantly higher CSF t-tau $(\mathrm{F}(1,57)=4.6, p=0.037), \mathrm{CSF} \operatorname{NFL}\left(\mathrm{F}(1,59)=155.5, p=3.53 \times 10^{-18}\right)$, and $\mathrm{VbV}$ $\left(\mathrm{F}(1,76)=34.6, p=1.03 \times 10^{-7}\right)$ than controls (Figure 1), with age and sex included as covariates. However the effect size for CSF $t$-tau is small (partial $\eta^{2}=0.07$ ), compared to large effects for $\mathrm{VbV}$ (partial $\eta^{2}=0.31$ ) and NFL (partial $\eta^{2}=0.72$ ). Logistic regression models were trained for each $\mathrm{N}$ metric, with age and sex included as covariates. 

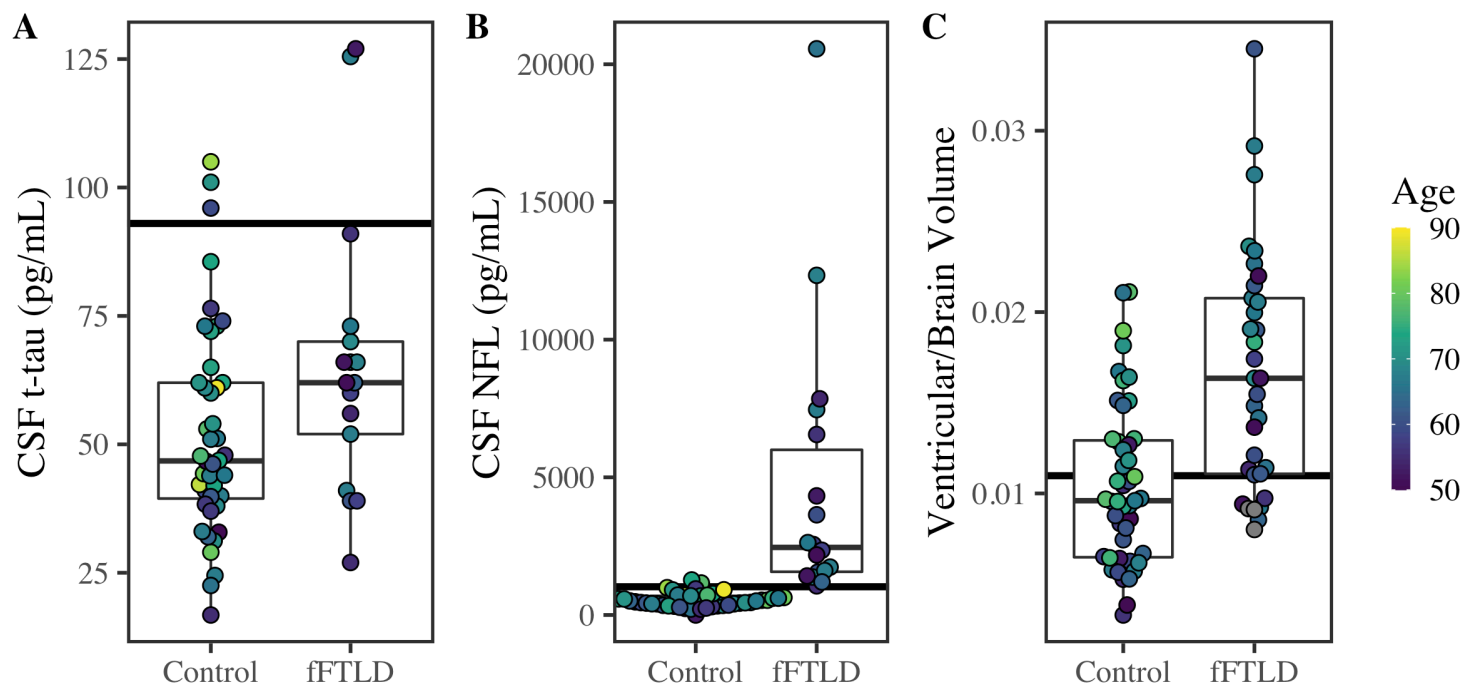

Figure 1. Boxplots of $\mathbf{N}$ measures in living Controls and fFTLD. CSF t-tau (Panel A), CSF NFL (Panel B) and VbV (Panel C) across Controls and fFTLD cases. Horizontal line indicates sample specific threshold as determined by ROC analyses and Youden's index. Color indicates age.

\section{Testing ATN classifications in autopsy cohort of AD and FTLD pathology}

Demographic Comparisons. AD patients were significantly older at CSF than FTLD, consistent with their older age of onset (Table 1), and also a longer interval from CSF to death than FTLD patients with CSF data. Unlike patients with CSF data, there was no difference in age at MRI or age at onset, disease duration or years of education between AD and FTLD with MRI data. Both CSF and MRI cohorts show that AD patients had a lower MMSE, lower CSF A $\beta_{1-42}$, higher CSF p-tau, and higher rates of co-pathology than FTLD. Both CSF and MRI cohorts show no difference between AD and FTLD patients for disease duration, years of education, or sex distribution.

ATN Classifications. We compared the classification of AD and FTLD patients without (AD, FTLD) and with (AD mixed, FTLD $\left._{\text {mixed }}\right)$ mixed pathology using each ATN strategy: ATN $_{\mathrm{TT}}$ (Table 3), ATN $\mathrm{AL}_{\mathrm{FL}}$ (Table 4), ATN $\mathrm{ATV}_{\mathrm{V}}$ (Table 5). ATN $\mathrm{FL}$ correctly identified 25 of 27 (93\%) FTLD cases as SNAP, and Fisher's tests confirm that this was significantly more 
than either $\operatorname{ATN}_{\mathrm{TT}}\left(30 \% ; \mathrm{OR}=27.3, \mathrm{CI}=5-290.6, p=3.09 \times 10^{-6}\right)$ or $\operatorname{ATN}_{\mathrm{VV}}(66 \% ; \mathrm{OR}=6.3$, $\mathrm{CI}=1.2-64.5, p=0.015)$. In addition, $\mathrm{ATN}$ Vv identified significantly more FTLD cases as SNAP than $\mathrm{ATN}_{\mathrm{TT}}(\mathrm{OR}=4.4, \mathrm{CI}=1.4-15.6, p=0.01)$.

Importantly, all 4 of the A+ FTLD cases from the CSF cohort were identified as concomitant SNAP and AD pathology $(\mathrm{A}+\mathrm{T}-\mathrm{N}+)$ by $\mathrm{ATN}_{\mathrm{FL}}$; this is compared to 1 of 4 identified by $\mathrm{ATN}_{\mathrm{TT}}$. For the 2 FTLD cases with AD co-pathology, $\mathrm{ATN}_{\mathrm{FL}}$ identified both as SNAP alone (A-T-N $\left.\mathrm{FL}^{+}\right)$, and $\mathrm{ATN}_{\mathrm{TT}}$ identified both as normal (A-T-N $\left.\mathrm{TT}^{-}\right)$. From the MRI cohort, ATNVV identified 3 of $5 \mathrm{~A}+$ FTLD cases as concomitant SNAP and AD pathology $\left(\mathrm{A}+\mathrm{T}-\mathrm{N}_{\mathrm{VV}}+\right)$; the other 2 were identified as $\mathrm{AD}$ pathologic change $\left(\mathrm{A}+\mathrm{T}-\mathrm{N}_{V^{-}}\right)$. Of note, in all A+ FTLD cases ( 7 total; CSF $n=2$; MRI n=3; Both CSF and MRI data $n=2$ ), AD severity was either low (CSF $n=3$; MRI $n=2)$ or not present (CSF $n=1 ;$ MRI $n=3)$, whereas the 2 FTLD cases with AD copathology (ADNPC=intermediate/high) were A(CSF $n=1$; CSF \& MRI data $n=1)$. These cases represent likely errors based on CSF A $\beta_{1}$ 42.

All three ATN strategies were equally likely to identify AD patients as Alzheimer's continuum disease, since this is determined by $\mathrm{A}+$ status $\left(\mathrm{CSF} A \beta_{1-42} \leq 192 \mathrm{pg} / \mathrm{mL}\right)$ for all three. $\mathrm{VbV}$ classified the largest proportion of $\mathrm{AD}$ patients as $\mathrm{N}+$, and Fisher's tests determined greater N-positivity than NFL $(\mathrm{OR}=5.8, \mathrm{CI}=1.4-28.8, p=0.0082)$ or $\mathrm{t}$-tau $(\mathrm{OR}=4.1, \mathrm{CI}=1-20.4, p=0.030)$. There was no difference in $\mathrm{N}$-positivity in AD across CSF NFL and t-tau $(p=0.46)$. 
Table 3: $A T N_{T T}$ classification of cases with negligible (AD, FTLD) and mixed (AD ${ }_{\text {mixed, }}$, FTLD mixed) co-pathology

\begin{tabular}{rrrrrr}
\hline Interpretation & Classification & AD & ADmixed & FTLD & FTLDmixed \\
\hline Normal & A-T-N $\mathrm{TT}^{-}$ & $1(3 \%)$ & $1(3 \%)$ & $14(64 \%)$ & $2(40 \%)$ \\
Alzheimer's pathologic change & $\mathrm{A}+\mathrm{T}-\mathrm{N}_{\mathrm{TT}^{-}}$ & $5(16 \%)$ & $10(27 \%)$ & $2(9 \%)$ & $1(20 \%)$ \\
$\mathrm{AD}$ & $\mathrm{A}+\mathrm{T}+\mathrm{N}_{\mathrm{TT}^{-}}$ & $9(28 \%)$ & $16(43 \%)$ & $0(0 \%)$ & $0(0 \%)$ \\
$\mathrm{AD}$ & $\mathrm{A}+\mathrm{T}+\mathrm{N}_{\mathrm{TT}^{+}}$ & $14(44 \%)$ & $7(19 \%)$ & $0(0 \%)$ & $0(0 \%)$ \\
SNAP and AD & $\mathrm{A}+\mathrm{T}-\mathrm{N}_{\mathrm{TT}^{+}}$ & $1(3 \%)$ & $1(3 \%)$ & $1(5 \%)$ & $0(0 \%)$ \\
SNAP & $\mathrm{A}-\mathrm{T}+\mathrm{N}_{\mathrm{TT}^{-}}$ & $1(3 \%)$ & $2(5 \%)$ & $0(0 \%)$ & $0(0 \%)$ \\
SNAP & $\mathrm{A}-\mathrm{T}-\mathrm{N}_{\mathrm{TT}^{+}}$ & $0(0 \%)$ & $0(0 \%)$ & $5(23 \%)$ & $1(20 \%)$ \\
SNAP & $\mathrm{A}-\mathrm{T}+\mathrm{N}_{\mathrm{TT}^{+}}$ & $1(3 \%)$ & $0(0 \%)$ & $0(0 \%)$ & $1(20 \%)$ \\
\hline
\end{tabular}

Table 4: $A T N_{F L}$ classification of cases with negligible (AD, FTLD) and mixed (AD ${ }_{\text {mixed }}$, FTLD ${ }_{\text {mixed }}$ ) co-pathology

\begin{tabular}{rrrrrr}
\hline Interpretation & Classification & $\mathrm{AD}$ & ADmixed & FTLD & FTLDmixed \\
\hline Normal & $\mathrm{A}-\mathrm{T}-\mathrm{N}_{\mathrm{FL}}-$ & $0(0 \%)$ & $1(3 \%)$ & $2(9 \%)$ & $0(0 \%)$ \\
Alzheimer's pathologic change & $\mathrm{A}+\mathrm{T}-\mathrm{N}_{\mathrm{FL}}{ }^{-}$ & $4(12 \%)$ & $8(22 \%)$ & $0(0 \%)$ & $0(0 \%)$ \\
$\mathrm{AD}$ & $\mathrm{A}+\mathrm{T}+\mathrm{N}_{\mathrm{FL}}-$ & $16(50 \%)$ & $18(49 \%)$ & $0(0 \%)$ & $0(0 \%)$ \\
$\mathrm{AD}$ & $\mathrm{A}+\mathrm{T}+\mathrm{N}_{\mathrm{FL}}+$ & $7(22 \%)$ & $5(14 \%)$ & $0(0 \%)$ & $0(0 \%)$ \\
SNAP and AD & $\mathrm{A}+\mathrm{T}-\mathrm{N}_{\mathrm{FL}}{ }^{+}$ & $2(6 \%)$ & $3(8 \%)$ & $3(14 \%)$ & $1(20 \%)$ \\
$\mathrm{SNAP}$ & $\mathrm{A}-\mathrm{T}+\mathrm{N}_{\mathrm{FL}}-$ & $2(6 \%)$ & $1(3 \%)$ & $0(0 \%)$ & $0(0 \%)$ \\
SNAP & $\mathrm{A}-\mathrm{T}-\mathrm{N}_{\mathrm{FL}}+$ & $1(3 \%)$ & $0(0 \%)$ & $17(77 \%)$ & $3(60 \%)$ \\
SNAP & $\mathrm{A}-\mathrm{T}+\mathrm{N}_{\mathrm{FL}}+$ & $0(0 \%)$ & $1(3 \%)$ & $0(0 \%)$ & $1(20 \%)$ \\
\hline
\end{tabular}

Table 5: $A T N_{v v}$ classification of cases with negligible (AD, FTLD) and mixed (AD mixed, FTLD $D_{\text {mixed }}$ ) co-pathology

\begin{tabular}{rrrrrr}
\hline Interpretation & Classification & $\mathrm{AD}$ & ADmixed & FTLD & FTLDmixed \\
\hline Normal & $\mathrm{A}-\mathrm{T}-\mathrm{N}_{\mathrm{VV}^{-}}$ & $0(0 \%)$ & $1(14 \%)$ & $10(32 \%)$ & $0(0 \%)$ \\
Alzheimer's pathologic change & $\mathrm{A}+\mathrm{T}-\mathrm{N}_{\mathrm{VV}}$ & $1(17 \%)$ & $0(0 \%)$ & $2(6 \%)$ & $0(0 \%)$ \\
$\mathrm{AD}$ & $\mathrm{A}+\mathrm{T}+\mathrm{N}_{\mathrm{VV}^{-}}$ & $1(17 \%)$ & $1(14 \%)$ & $0(0 \%)$ & $0(0 \%)$ \\
$\mathrm{AD}$ & $\mathrm{A}+\mathrm{T}+\mathrm{N}_{\mathrm{VV}}+$ & $3(50 \%)$ & $4(57 \%)$ & $0(0 \%)$ & $0(0 \%)$ \\
SNAP and AD & $\mathrm{A}+\mathrm{T}-\mathrm{N}_{\mathrm{VV}^{+}}$ & $1(17 \%)$ & $1(14 \%)$ & $2(6 \%)$ & $1(25 \%)$ \\
$\mathrm{SNAP}$ & $\mathrm{A}-\mathrm{T}+\mathrm{N}_{\mathrm{VV}^{-}}$ & $0(0 \%)$ & $0(0 \%)$ & $0(0 \%)$ & $1(25 \%)$ \\
SNAP & $\mathrm{A}-\mathrm{T}-\mathrm{N}_{\mathrm{VV}}+$ & $0(0 \%)$ & $0(0 \%)$ & $16(52 \%)$ & $2(50 \%)$ \\
SNAP & $\mathrm{A}-\mathrm{T}+\mathrm{N}_{\mathrm{VV}}+$ & $0(0 \%)$ & $0(0 \%)$ & $1(3 \%)$ & $0(0 \%)$ \\
\hline
\end{tabular}




\section{Comparisons of $N$ across $A D$ and FTLD-TDP and FTLD-tau}

Figure 2 illustrates differences in CSF t-tau, CSF NFL and VbV across AD, FTLD-TDP, and FTLD-tau. ANCOVAs indicated that both CSF t-tau $(\mathrm{F}(2,88)=7.5, p=0.001)$ and CSF $\operatorname{NFL}\left(\mathrm{F}(2,87)=21, p=3.52 \times 10^{-8}\right)$ significantly differed across pathologies, while VbV did not $(p=0.75)$. Post-hoc tests confirmed that CSF t-tau was significantly lower in FTLDTDP than $\operatorname{AD}\left(\beta=-0.81, p=8.0 \times 10^{-4}\right)$; there was no difference between AD and FTLD-tau $(p=0.06)$ or between FTLD-TDP and FTLD-tau $(p=0.38)$. Post-hoc tests also confirmed that CSF NFL was significantly higher in FTLD-TDP $\left(\beta=0.013, p=2.06 \times 10^{-8}\right)$ and FTLDtau $\left(\beta=0.008, p=9.0 \times 10^{-4}\right)$ than AD; CSF NFL did not significantly differ in FTLD-TDP compared to FTLD-tau $(\beta=-0.005, p=0.07)$. Because concentrations of CSF NFL are generally higher in ALS than other forms of FTLD ${ }^{28,45}$, we repeated NFL analyses excluding FTLD-ALS cases. Results were consistent, with significantly higher CSF NFL in FTLD-TDP $\left(\beta=0.012, p=5.96 \times 10^{-7}\right)$ and FTLD-tau $\left(\beta=0.008, p=3.88 \times 10^{-4}\right)$ than AD; there was no difference between FTLD-TDP and FTLD-tau $(p=0.24)$.

All ANCOVAs included the covariates of co-pathology, age, interval to death, sex, and MMSE. Co-pathology (t-tau, $p=0.69$; NFL, $p=0.92$; VbV $p=0.6$ ) was not significant in any of the models. Interval to death was negatively associated with $\operatorname{NFL}(\mathrm{F}(1,87)=5.3, p=0.02)$, but not t-tau $(p=0.73)$ or $\mathrm{VbV}(p=0.21)$. Age was positively associated with NFL $(\mathrm{F}(1,87)=10.1, p=0.002)$ as seen previously ${ }^{17}$, but not with $\mathrm{t}$-tau $(p=0.96)$ or $\operatorname{VbV}(p=0.19)$. Males had significantly lower t-tau levels than females $(\mathrm{F}(1,88)=5.68, p=0.02)$, but sex was not a significant factor for NFL levels $(p=0.44)$ or $\operatorname{VbV}(p=0.108)$. Finally, MMSE was negatively associated with $\mathrm{NFL}(\mathrm{F}(1,87)=4.3, p=0.04)$, but not $\mathrm{t}$-tau $(p=0.26)$ or $\mathrm{VbV}$ 
$(p=0.4)$. Neither scanner $(p=0.19)$ nor pulse sequence $(p=0.32)$ were significant factors for $\mathrm{VbV}$.
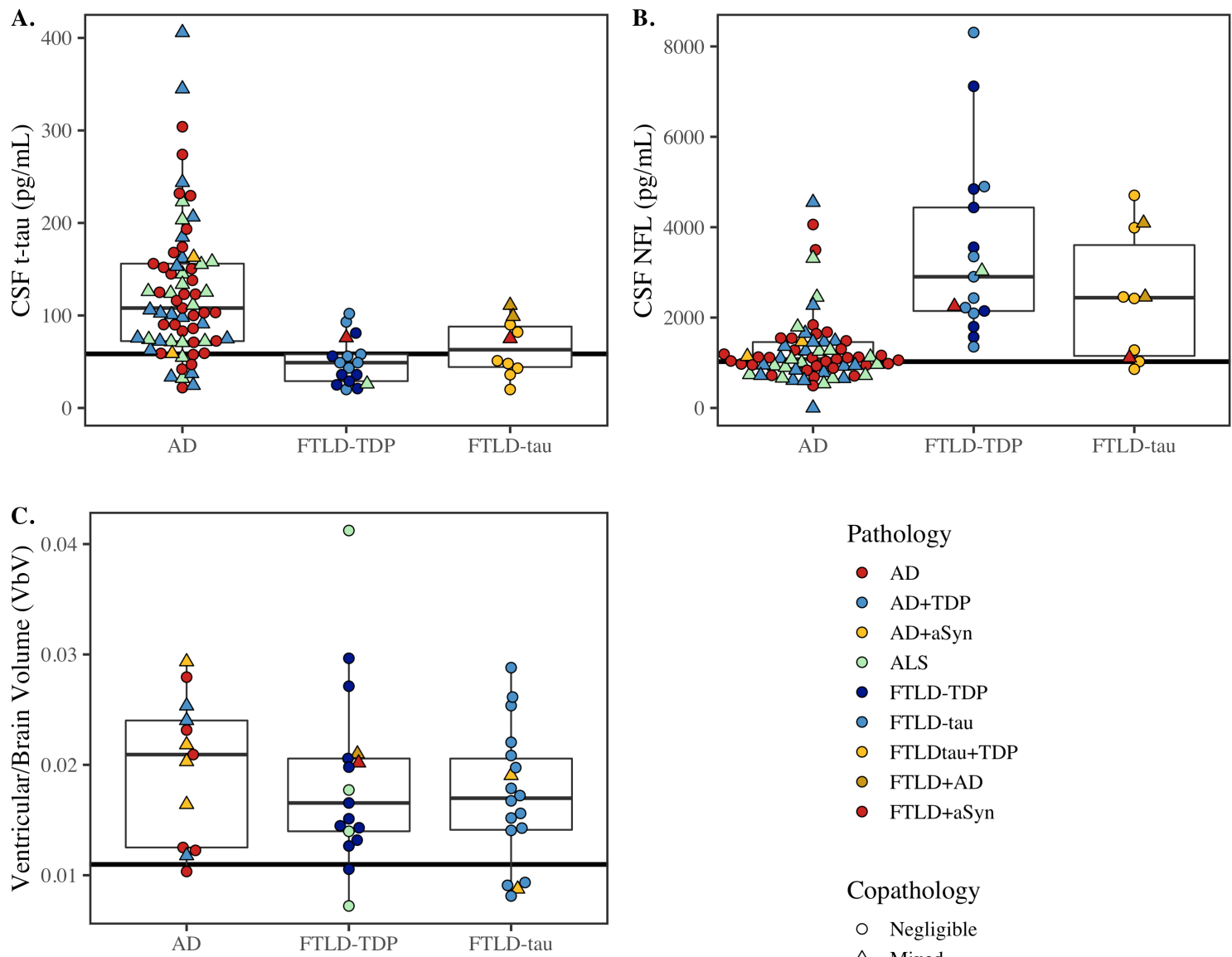

$$
\begin{aligned}
& \text { Pathology } \\
& \text { - } \mathrm{AD} \\
& \text { - } A D+T D P \\
& \text { - AD+aSyn } \\
& \text { - ALS } \\
& \text { - FTLD-TDP } \\
& \text { - FTLD-tau } \\
& \text { - FTLDtau+TDP } \\
& \text { - FTLD+AD } \\
& \text { - FTLD+aSyn } \\
& \text { Copathology } \\
& \text { ○ Negligible } \\
& \triangle \text { Mixed }
\end{aligned}
$$

Figure 2. Boxplots of $\mathbf{N}$ in autopsy-confirmed AD, FTLD-TDP and FTLD-tau. CSF ttau (Panel A), CSF NFL (Panel B) and VbV (Panel C) across pathology groups. Horizontal line indicates sample specific threshold as determined by ROC analyses and Youden's index. Shape indicates presence of co-pathology. Color indicates primary pathological subtype/co-pathological presence of AD (red), TDP (blue; ALS in dark blue), tau (yellow; co-occurring tau and TDP in dark yellow), or $\alpha$-synuclein (aSyn; light green).

Because $\mathrm{ATN}_{\mathrm{FL}}$ had the best performance when identifying FTLD as SNAP, we performed a follow-up analysis of $\mathrm{N}_{\mathrm{FL}}$-status in $\mathrm{AD}$. A linear model (Equation 4) tested NFL as a prognostic indicator in $\mathrm{AD}$, and how it related to cognitive impairment (MMSE). A logistic model (Equation 5) examined factors that predicted $\mathrm{N}_{\mathrm{FL}}$ status $\left(\mathrm{N}_{\mathrm{FL}}+=1 ; \mathrm{N}_{\mathrm{FL}}-\right.$ 
$=0)$ in $\mathrm{AD}$. Because education interacts with cognitive decline ${ }^{46}$, we included years of education as a factor in both models. We also tested if $\mathrm{ABC}$ score (intermediate, high) related to MMSE or $\mathrm{N}_{\mathrm{FL}}$ status in $\mathrm{AD}$.

[4] MMSE $\sim-\mathrm{NFL}^{-1 / 2}+$ Co-pathology + Age at CSF + CSF to MMSE interval $+\mathrm{Sex}+\mathrm{ABC}$ + Education

[5] $\mathrm{P}\left(\mathrm{N}_{\mathrm{FL}}+\right) \sim$ Co-pathology + Age at CSF $+\mathrm{Sex}+\mathrm{ABC}+$ Education $+\mathrm{MMSE}$

MMSE was negatively associated with NFL $(\beta=-335.1, \mathrm{SE}=103.6, p=0.002)$ in $\mathrm{AD}$; copathology $(p=0.19)$, age $(\beta=0.2, \mathrm{SE}=0.08, p=0.012), \mathrm{CSF}$ to MMSE interval $(p=0.085)$, sex $(p=0.388), \mathrm{ABC}$ scores $(p=0.15)$, and education $(\beta=0.6, \mathrm{SE}=0.2, p=0.004)$ were included as covariates. Neither CSF t-tau nor $\mathrm{VbV}$ was related to MMSE $(p>0.2)$. The logistic regression showed that only MMSE predicted $\mathrm{N}_{\mathrm{FL}}$-status in $\mathrm{AD}(\beta=-0.13, \mathrm{SE}=0.06$, $p=0.046$ ). All other factors were not significant (all $p>0.3$ ); we note that the model used to

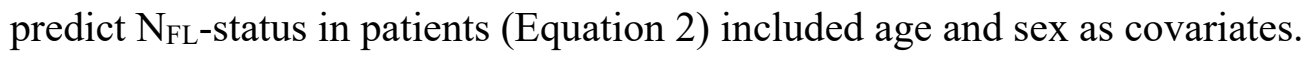

\section{Diagnostic comparisons}

ROC analyses compared diagnostic accuracy of each biomarker when discriminating fFTLD from controls (Table 6A) and when discriminating FTLD from AD (Table 6B). In the independent cohort, NFL had the best performance discriminating fFTLD from controls. Comparing two correlated ROC curves for CSF analytes with DeLong's test, we found that NFL had a significantly higher AUC than t-tau $\left(Z=4.2, p=2.49 \times 10^{-5}\right), A \beta_{1-42}(Z=6.5$, $\left.p=7.30 \times 10^{-11}\right)$, and $\mathrm{p}$-tau $\left(\mathrm{Z}=3.9, p=1.39 \times 10^{-4}\right)$ when discriminating fFTLD patients from controls (Table 6A). In the autopsy cohort, CSF $A \beta_{1-42}$ had the best performance discriminating FTLD from AD. 
Table 6. ROC analyses for all biomarkers. Area under the curve (AUC), and sensitivity and specificity at best threshold when discriminating A.) fFTLD from controls, and B.) FTLD pathology from AD pathology.

\section{A. Independent cohort}

\begin{tabular}{lrrrr}
\hline Measure & AUC & Threshold & Sensitivity & Specificity \\
\hline $\mathrm{A} \beta_{1-42}$ & 0.46 & 261.00 & 0.61 & 0.50 \\
p-tau & 0.72 & 16.50 & 0.83 & 0.72 \\
t-tau & 0.66 & 51.58 & 0.76 & 0.61 \\
NFL & 1.00 & 1025.17 & 1.00 & 0.96 \\
VbV & 0.78 & 0.01 & 0.79 & 0.64 \\
\hline
\end{tabular}

\section{B. Autopsy Cohort}

\begin{tabular}{lrrrr}
\hline Measure & AUC & Threshold & Sensitivity & Specificity \\
\hline $\mathrm{A} \beta_{1-42}$ & 0.94 & 196.31 & 0.85 & 0.93 \\
p-tau & 0.93 & 20.34 & 0.96 & 0.83 \\
t-tau & 0.83 & 58.46 & 0.67 & 0.87 \\
NFL & 0.87 & 1796.38 & 0.78 & 0.90 \\
VbV & 0.60 & 0.02 & 0.71 & 0.62 \\
\hline
\end{tabular}

\section{Discussion}

$(1,556 / 1,500$ words $)$

In an autopsy-confirmed cohort of patients with AD and FTLD pathology, we tested ATN classifications using CSF t-tau as a traditional marker of $\mathrm{N}$ ( $\mathrm{ATN}_{\mathrm{TT}}$ ) compared to two modified frameworks (ATN $\mathrm{FL}_{\mathrm{FL}} \mathrm{ATN} \mathrm{NV}_{\mathrm{V}}$ ) using $\mathrm{CSF}$ NFL and $\mathrm{VbV}$ as alternative markers of N. Because all three frameworks defined A and T status using CSF A $\beta_{1-42}$ and CSF ptau, identification of $\mathrm{AD}$ pathologic cases as Alzheimer's continuum disease $(\mathrm{A}+)$ is equivalent. ATN $\mathrm{FL}_{\mathrm{L}}$ was highly sensitive to primary pathology in both $\mathrm{AD}$ and FTLD diseases, classifying $91 \%$ of AD patients as Alzheimer's spectrum disease and $93 \%$ of FTLD patients as SNAP. When classifying patients with primary FTLD pathology, $\mathrm{ATN}_{\mathrm{FL}}$ was more accurate than ATNVv (66\% of FTLD as SNAP) or ATNTT $(30 \%$ of FTLD as SNAP). 
In agreement with previous findings ${ }^{28,47,48}$, we found that NFL was higher in FTLD than AD, including both FTLD-TDP and FTLD-tau. We observed marginal or no differences in FTLD-TDP or FTLD-tau for t-tau, NFL, or VbV. While NFL, t-tau, and VbV levels of N were elevated in fFTLD compared with controls, the effect size was largest for NFL, explaining the high accuracy of ATNFL when identifying FTLD as SNAP. Our models indicate that the presence/absence of secondary pathologies was not a significant factor for t-tau, NFL, or VbV levels. Sex was a significant factor for t-tau concentrations, while age, interval to death and MMSE were significant factors for NFL concentrations.

Given the relative added value of NFL in the ATN framework compared to t-tau, we suggest that NFL alone is sufficient to distinguish AD from FTLD. However, NFL $(\mathrm{AUC}=0.87)$ is less accurate than $\mathrm{A} \beta_{1-42}(\mathrm{AUC}=0.94)$ or $\mathrm{p}$-tau $(\mathrm{AUC}=0.93)$, which consistently show excellent discrimination ${ }^{13,14,47}$, and is only somewhat higher than t-tau $(\mathrm{AUC}=0.83)$. Unlike $A \beta_{1-42}$ and $\mathrm{p}$-tau, which are $\mathrm{AD}$-specific markers and discriminate AD from both FTLD and healthy controls ${ }^{13,14,47}$, our results show that NFL was nearly perfect when discriminating fFTLD from cognitively unimpaired controls (1.00 sensitivity and 0.96 specificity); $\mathrm{VbV}$ was fair $(\mathrm{AUC}=0.78)$ and $\mathrm{t}$-tau performed poorly $(\mathrm{AUC}=0.66)$. These results highlight that an important value of $\mathrm{N}$ as a marker of degeneration is discriminating SNAP from healthy controls.

In addition to detection of FTLD as SNAP, we also investigated $\mathrm{N}_{\mathrm{FL}}$ status in AD. While higher concentrations of NFL are found in FTLD associated diseases ${ }^{28,47,48}$, NFL has also been shown to be a prognostic indicator of disease severity and survival in $\mathrm{AD} 17,18,20$. Another study comparing CSF NFL levels by $\mathrm{ATN}_{\mathrm{TT}}$ profiles in living controls and dementia patients found elevated NFL in tau positive groups $(\mathrm{T}+$ and/or $\mathrm{N}+)$ compared to 
tau negative (A+T-N-, A-T-N-), suggesting that NFL levels may be higher in AD patients with more severe disease staging ${ }^{49}$. In this study we also see evidence that higher NFL concentrations relate to disease severity in AD; lower MMSE scores were associated with higher NFL levels in $\mathrm{AD}$, and also predicted $\mathrm{N}_{\mathrm{FL}}+$ status in $\mathrm{AD}$.

A potential strength of the ATN framework is its aim to identify cases with likely concomitant $\mathrm{AD}$ and SNAP $(\mathrm{A}+\mathrm{T}-\mathrm{N}+)$. While co-occurring proteinopathies are common $^{11,50}$, binary stratification by $\mathrm{AD}$ biomarkers - $\mathrm{AD}$ or not $\mathrm{AD}$ - does not capture this pathologic heterogeneity. Indeed, cases with primary SNAP and secondary AD pathology are likely to have positive AD biomarkers ${ }^{2,12}$, obscuring the presence of SNAP. Such misclassifications can increase noise in clinical treatment trials, since these cases might not respond to $\mathrm{AD}$-specific pharmacological targets. By combining markers of $\mathrm{AD}$ pathological hallmarks (A and T) with N, the ATN framework provides a strategy to detect cases with likely concomitant SNAP and AD. Even so, our results show that 3 of 4 FTLD cases were misdiagnosed by $\mathrm{ATN}_{\mathrm{TT}}$ as $\mathrm{AD}$ alone (A+T-N-), missing the primary pathology of FTLD. By comparison, ATN $\mathrm{FL}_{\mathrm{FL}}$ classified all 4 of $4 \mathrm{~A}+\mathrm{FTLD}$ CSF cases as concomitant $\mathrm{AD}$ and $\mathrm{SNAP}\left(\mathrm{A}+\mathrm{T}-\mathrm{N}_{\mathrm{FL}}+\right) ; \mathrm{ATN} \mathrm{VV}_{\mathrm{V}}$ classified 3 of $5 \mathrm{~A}+\mathrm{FTLD}$ MRI cases as concomitant $\mathrm{AD}$ and SNAP. This is a small number of cases, so we can only modestly speculate about a more generalized application of this finding. Still, results indicate that $\mathrm{ATN}_{\mathrm{FL}}$ was sensitive to primary FTLD pathology with A+ status. This was not the case for AD cases with secondary FTLD-tau: 1 of 3 was $\mathrm{A}+\mathrm{T}-\mathrm{N}_{\mathrm{FL}}+$ and the other two were $\mathrm{N}_{\mathrm{FL}}-\left(\mathrm{A}+\mathrm{T}+\mathrm{N}_{\mathrm{FL}}-\right.$ and $\mathrm{A}+\mathrm{T}-\mathrm{N}_{\mathrm{FL}}-$ ). Indeed, all three $\mathrm{ATN}$ strategies classified the large majority of $\mathrm{AD}_{\text {mixed }}$ as something other than concomitant AD and SNAP $(\mathrm{A}+\mathrm{T}-\mathrm{N}+)$. Thus, no strategy reliably detected co-pathology when AD was the primary pathology. 
While $\mathrm{ATN}_{\mathrm{FL}}$ had the best performance at identifying FTLD patients as SNAP (93\%), we note that ATNVv also classified a higher percentage of FTLD patients as SNAP $(66 \%)$ than $\operatorname{ATN}_{\mathrm{TT}}(30 \%)$. While AD-specific or FTLD-specific signatures of atrophy can accurately discriminate pathologies ${ }^{51-53}$, we tested $\mathrm{VbV}$ as a global marker of degeneration that is not specific to $\mathrm{AD}$ and that may be adequate to capture the heterogeneous atrophy patterns found in $\mathrm{AD}$ and FTLD ${ }^{8,54,55}$. Indeed, both $\mathrm{AD}$ and FTLD patients show ventricular enlargement and shrinking total brain volume with disease progression ${ }^{21,22,24,25}$. Our results support $\mathrm{VbV}$ as a potential marker of $\mathrm{N}$ that is not specific to $\mathrm{AD}$; $\mathrm{VbV}$ was significantly elevated in fFTLD compared to controls, showed no differences across pathologies (AD, FTLD), and ATNvv was fair at classifying AD and FTLD patients. An important caveat to these results is that a smaller subset of AD patients had MRI scans, and that the AD MRI cohort was significantly younger at onset and more impaired as measured by MMSE than the AD CSF cohort. Thus, this study can suggest only limited conclusions about the accuracy of $\mathrm{VbV}$ as a marker of $\mathrm{N}$ compared to CSF t-tau or NFL, or regarding $\mathrm{VbV}$ differences across proteinopathies.

We do note some important limitations to $\mathrm{ATN} \mathrm{FL}_{\mathrm{FL}}$. In terms of likely errors, $\mathrm{ATN} \mathrm{NL}_{\mathrm{FL}}$ classified 5 of 69 AD patients as SNAP and 3 patients as normal (1 AD; 2 FTLD). While NFL was sensitive to primary FTLD pathology, it was not an indicator of mixed pathology in $\mathrm{AD}$. A minority of $\mathrm{AD}_{\text {mixed }}$ cases were $\mathrm{N}_{\mathrm{FL}}+(26 \%)$; more specifically, of the 3 cases of AD with secondary FTLD-tau, only 1 was $\mathrm{N}_{\mathrm{FL}}+$. Designation of $\mathrm{AD}_{\text {mixed }}$ as concomitant $\mathrm{AD}$ and SNAP $\left(\mathrm{A}+\mathrm{T}-\mathrm{N}_{\mathrm{FL}}+\right)$ was low $(8 \%)$, and equivalent to $\mathrm{AD}$ with negligible copathology $(6 \%)$. This highlights an important caveat: we do not expect $\mathrm{N}_{\mathrm{FL}}$ to generalize to all proteinopathies, some of which have relatively low concentrations of NFL ${ }^{16,28}$. Our 
results thus indicate that $\mathrm{AD}$ patients with secondary pathologies like $\alpha$-synuclein, LATE, or hippocampal sclerosis may not be $\mathrm{N}_{\mathrm{FL}}+$. Conversely, other diseases with severe axonal damage, including vascular disease, are also likely to be $\mathrm{N}_{\mathrm{FL}}+{ }^{16,17}$. Thus, interpretation of ATN $\mathrm{FL}_{\mathrm{FL}}$ status may require consideration of other diagnostic hallmarks, such as the presence of parkinsonian motor features, FTLD-associated clinical features, or white matter hyperintensities on MRI. As informative biomarkers are developed for additional conditions, these may be combined with $\mathrm{ATN}_{\mathrm{FL}}$ to provide more accurate and specific diagnoses within a heterogeneous population.

There are additional caveats to our findings. First, this was a retrospective study with limited data availability, and we used different cohorts to evaluate CSF metrics (t-tau, NFL) and MRI metrics $(\mathrm{VbV})$. The sample size for the AD MRI cohort was particularly small and demographically different from the AD CSF cohort. Consequently, we could not test rate of $\mathrm{VbV}$ change as a marker of degeneration, and we may have lacked power to detect factors related to $\mathrm{N}_{\mathrm{Vv}}$ status in $\mathrm{AD}$. Second, different scanners and pulse sequences were used to acquire MRI data, and although we included these as covariates in our logistic and ANCOVA models, the lack of a standardized protocol may have added variance and prevented us from detecting true differences across factors, including pathology. Third, while a strength of this study was the use of an independent living sample to determine $\mathrm{N}_{\mathrm{TT}}, \mathrm{N}_{\mathrm{FL}}$ and $\mathrm{N}_{\mathrm{Vv}}$ status, without autopsy-confirmation it is possible that the cognitively unimpaired controls had undetected pathologic conditions, or that some fFTLD patients had mixed pathologies. Finally, we cannot be certain of the pathological status of our autopsy patients at the time that CSF/MRI was obtained during life, and it could be that some cases were true-negatives for neurodegeneration at time of observation. To account 
for this confound, we examined how the interval to death related to each $\mathrm{N}$ metric, and included only symptomatic patients in fFTLD and autopsy cohorts. While we assume that most patients are $\mathrm{N}+$, this cannot be confirmed, and with no standardized method to compare severity of neurodegeneration across a heterogeneous population, we are unable to assess how $\mathrm{N}$ status in $\mathrm{AD}$ and FTLD relates to degree of degeneration.

In summary, we find that NFL is a highly accurate marker of N-status that is both highly sensitive to FTLD pathology and is indicative of cognitive impairment in AD. ATN $N_{F L}$ had the best classification performance across all three ATN strategies, correctly identifying 91\% of AD patients as having Alzheimer's spectrum disease and 93\% of FTLD patients as SNAP. 


\section{Acknowledgements}

This work was supported in part by NIH AG017586, AG052943, AG054519, AG066597, AG10124. HZ is a Wallenberg Scholar supported by grants from the Swedish Research Council (\#2018-02532), the European Research Council (\#681712), Swedish State Support for Clinical Research (\#ALFGBG-720931), the Alzheimer Drug Discovery Foundation (ADDF), USA (\#201809-2016862), and the UK Dementia Research Institute at UCL. KB

is supported by the Swedish Research Council (\#2017-00915), the Alzheimer Drug Discovery Foundation (ADDF), USA (\#RDAPB-201809-2016615), the Swedish Alzheimer Foundation (\#AF-742881), Hjärnfonden, Sweden (\#FO2017-0243), the Swedish state under the agreement between the Swedish government and the County Councils, the ALF-agreement (\#ALFGBG-715986), and European Union Joint Program for Neurodegenerative Disorders (JPND2019-466-236). KAQ Cousins is a recipient of the Alzheimer's Association Research Fellowship to Promote Diversity (AARF-D).

Author Contributions

Conception and design of the study: KAQC, MG, JSP, DJI, CTM

Data acquisition: DJI, DAW, LMJS, EBL, JQT, MG, GSG, NK, SB

Data analysis: KAQC

Manuscript drafting: KAQC, MG, JSP, DJI, CTM, EBL, DAW, LMJS, JQT, SB, NK, GSG, $\mathrm{HZ}, \mathrm{KB}$

\section{Potential Conflicts of Interest}

HZ has served at scientific advisory boards for Denali, Roche Diagnostics, Wave, Samumed and $\operatorname{CogRx}$, has given lectures in symposia sponsored by Fujirebio, Alzecure and Biogen, and is a co-founder of Brain Biomarker Solutions in Gothenburg AB (BBS), 
which is a part of the GU Ventures Incubator Program. KB has served as a consultant, at advisory boards, or at data monitoring committees for Abcam, Axon, Biogen, Julius Clinical, Lilly, MagQu, Novartis, Roche Diagnostics, and Siemens Healthineers, and is a co-founder of Brain Biomarker Solutions in Gothenburg AB (BBS), which is a part of the GU Ventures Incubator Program. KAQC has no conflicts of interest to report. 


\section{References}

1. Jack CR, Bennett DA, Blennow K, et al. NIA-AA Research Framework: Toward a biological definition of Alzheimer's disease [Internet]. Alzheimer's Dement. 2018;14(4):535-562.Available from: https://doi.org/10.1016/j.jalz.2018.02.018

2. Cousins KAQ, Irwin DJ, Wolk DA, et al. ATN status in amnestic and nonamnestic Alzheimer's disease and frontotemporal lobar degeneration. bioRxiv 2019;

3. Soldan A, Pettigrew C, Fagan AM, et al. ATN profiles among cognitively normal individuals and longitudinal cognitive outcomes. Neurology 2019;92(14):e1567e1579.

4. Haense C, Buerger K, Kalbe E, et al. CSF total and phosphorylated tau protein, regional glucose metabolism and dementia severity in Alzheimer's disease. Eur. J. Neurol. 2008;15(11):1155-1162.

5. Diniz BSO, Pinto Jr JA, Forlenza OV. Do CSF total tau, phosphorylated tau, and $\beta$-amyloid 42 help to predict progression of mild cognitive impairment to Alzheimer's disease? A systematic review and meta-analysis of the literature. World J. Biol. Psychiatry 2008;9(3):172-182.

6. Landau SM, Harvey D, Madison CM, et al. Comparing predictors of conversion and decline in mild cognitive impairment. Neurology 2010;75(3):230-238.

7. Olsson B, Lautner R, Andreasson U, et al. CSF and blood biomarkers for the diagnosis of Alzheimer's disease: a systematic review and meta-analysis. Lancet Neurol. 2016;15(7):673-684.

8. Ekman U, Ferreira D, Westman E. The A/T/N biomarker scheme and patterns of brain atrophy assessed in mild cognitive impairment. Sci. Rep. 2018;8(1):1-10.

9. Khoonsari PE, Shevchenko G, Herman S, et al. Improved differential diagnosis of Alzheimer's disease by integrating ELISA and mass spectrometry-based cerebrospinal fluid biomarkers. J. Alzheimer's Dis. 2019;67(2):639-651.

10. Vos SJB, Duara R. The prognostic value of ATN Alzheimer biomarker profiles in cognitively normal individuals. 2019;

11. Robinson JL, Lee EB, Xie SX, et al. Neurodegenerative disease concomitant proteinopathies are prevalent, age-related and APOE4-associated. Brain 2018;141(7):2181-2193.

12. Toledo JB, Brettschneider J, Grossman M, et al. CSF biomarkers cutoffs: the importance of coincident neuropathological diseases. Acta Neuropathol. 2012;124(1):23-35.

13. Skillbäck T, Farahmand BY, Rosen C, et al. Cerebrospinal fluid tau and amyloid$\beta 1-42$ in patients with dementia. Brain 2015;138(9):2716-2731. 
14. Grossman M, Farmer J, Leight S, et al. Cerebrospinal fluid profile in frontotemporal dementia and Alzheimer's disease. Ann. Neurol. 2005;57(5):721729.

15. De Jong D, Jansen R, Pijnenburg YAL, et al. CSF neurofilament proteins in the differential diagnosis of dementia. J. Neurol. Neurosurg. Psychiatry 2007;78(9):936-938.

16. Bridel C, Van Wieringen WN, Zetterberg H, et al. Diagnostic value of cerebrospinal fluid neurofilament light protein in neurology: a systematic review and meta-analysis. JAMA Neurol. 2019;76(9):1035-1048.

17. Skillbäck T, Farahmand B, Bartlett JW, et al. CSF neurofilament light differs in neurodegenerative diseases and predicts severity and survival. Neurology 2014;83(21):1945-1953.

18. Kern S, Syrjanen JA, Blennow K, et al. Association of cerebrospinal fluid neurofilament light protein with risk of mild cognitive impairment among individuals without cognitive impairment. JAMA Neurol. 2019;76(2):187-193.

19. Scherling CS, Hall T, Berisha F, et al. Cerebrospinal fluid neurofilament concentration reflects disease severity in frontotemporal degeneration. Ann. Neurol. 2014;75(1):116-126.

20. Zetterberg H, Skillbäck T, Mattsson N, et al. Association of cerebrospinal fluid neurofilament light concentration with Alzheimer disease progression. JAMA Neurol. 2016;73(1):60-67.

21. Silbert LC, Quinn JF, Moore MM, et al. Changes in premorbid brain volume predict Alzheimer's disease pathology. Neurology 2003;61(4):487-492.

22. Nestor SM, Rupsingh R, Borrie M, et al. Ventricular enlargement as a possible measure of Alzheimer's disease progression validated using the Alzheimer's disease neuroimaging initiative database. Brain 2008;131(9):2443-2454.

23. Carmichael OT, Kuller LH, Lopez OL, et al. Ventricular volume and dementia progression in the Cardiovascular Health Study. Neurobiol. Aging 2007;28(3):389-397.

24. Tavares TP, Mitchell DG V, Coleman K, et al. Ventricular volume expansion in presymptomatic genetic frontotemporal dementia. Neurology 2019;93(18):e1699_ e1706.

25. Knopman DS, Jack CR, Kramer JH, et al. Brain and ventricular volumetric changes in frontotemporal lobar degeneration over 1 year. Neurology 2009;72(21):1843-1849.

26. Xie SX, Baek Y, Grossman M, et al. Building an integrated neurodegenerative disease database at an academic health center [Internet]. Alzheimer's Dement. 2011;7(4):e84-e93.Available from: http://dx.doi.org/10.1016/j.jalz.2010.08.233 
27. Toledo JB, Van Deerlin VM, Lee EB, et al. A platform for discovery: the University of Pennsylvania integrated neurodegenerative disease biobank. Alzheimer's Dement. 2014;10(4):477-484.

28. Olsson B, Portelius E, Cullen NC, et al. Association of cerebrospinal fluid neurofilament light protein levels with cognition in patients with dementia, motor neuron disease, and movement disorders. JAMA Neurol. 2019;76(3):318-325.

29. Igaz LM, Kwong LK, Xu Y, et al. Enrichment of C-terminal fragments in TAR DNA-binding protein-43 cytoplasmic inclusions in brain but not in spinal cord of frontotemporal lobar degeneration and amyotrophic lateral sclerosis. Am. J. Pathol. 2008;173(1):182-194.

30. Mackenzie IRA, Neumann M, Bigio EH, et al. Nomenclature and nosology for neuropathologic subtypes of frontotemporal lobar degeneration: an update. Acta Neuropathol. 2010;119(1):1.

31. Montine TJ, Phelps CH, Beach TG, et al. National institute on aging-Alzheimer's association guidelines for the neuropathologic assessment of Alzheimer's disease: A practical approach. Acta Neuropathol. 2012;123(1):1-11.

32. Gibbons GS, Banks RA, Kim B, et al. Detection of Alzheimer disease (AD)specific tau pathology in $\mathrm{AD}$ and non $\mathrm{AD}$ tauopathies by immunohistochemistry with novel conformation-selective tau antibodies. J. Neuropathol. Exp. Neurol. 2018;77(3):216-228.

33. Gibbons GS, Kim SJ, Robinson JL, et al. Detection of Alzheimer's disease (AD) specific tau pathology with conformation-selective anti-tau monoclonal antibody in co-morbid frontotemporal lobar degeneration-tau (FTLD-tau). Acta Neuropathol. Commun. 2019;7(1):34.

34. McKeith IG, Dickson DW, Lowe J, et al. Diagnosis and management of dementia with Lewy bodies: Third report of the DLB consortium. Neurology 2005;65(12):1863-1872.

35. Folstein MF, Folstein SE, McHugh PR. "Mini-mental state". A practical method for grading the cognitive state of patients for the clinician [Internet]. J. Psychiatr. Res. 1975;12(3):189-198.Available from: http://linkinghub.elsevier.com/retrieve/pii/0022395675900266

36. Woollacott IOC, Rohrer JD. The clinical spectrum of sporadic and familial forms of frontotemporal dementia. J. Neurochem. 2016;138:6-31.

37. Borroni B, Agosti C, Premi E, et al. The FTLD-modified Clinical Dementia Rating scale is a reliable tool for defining disease severity in Frontotemporal Lobar Degeneration: evidence from a brain SPECT study. Eur. J. Neurol. 2010;17(5):703-707.

38. Shaw LM, Vanderstichele H, Knapik-Czajka M, et al. Qualification of the analytical and clinical performance of CSF biomarker analyses in ADNI. Acta 
Neuropathol. 2011;121(5):597-609.

39. Shaw LM, Vanderstichele H, Knapik-Czajka M, et al. Cerebrospinal fluid biomarker signature in alzheimer's disease neuroimaging initiative subjects. Ann. Neurol. 2009;65(4):403-413.

40. Tustison NJ, Cook PA, Klein A, et al. Large-scale evaluation of ANTs and FreeSurfer cortical thickness measurements. Neuroimage 2014;99:166-179.

41. R Core Team. R: A language and environment for statistical computing. [Internet]. 2017;Available from: https://www.r-project.org/

42. Fox J, Weisberg S, Adler D, et al. Package 'car.' Vienna R Found. Stat. Comput. 2012;

43. Hothorn T, Bretz F, Westfall P. Simultaneous inference in general parametric models. Biometrical J. J. Math. Methods Biosci. 2008;50(3):346-363.

44. Robin X, Turck N, Hainard A, et al. pROC: an open-source package for R and S+ to analyze and compare ROC curves. BMC Bioinformatics 2011;12(1):77.

45. Forgrave LM, Ma M, Best JR, DeMarco ML. The diagnostic performance of neurofilament light chain in CSF and blood for Alzheimer's disease, frontotemporal dementia, and amyotrophic lateral sclerosis: A systematic review and meta-analysis. Alzheimer's Dement. Diagnosis, Assess. Dis. Monit. 2019;11:730-743.

46. Crum RM, Anthony JC, Bassett SS, Folstein MF. Population-based norms for the Mini-Mental State Examination by age and educational level. Jama 1993;269(18):2386-2391.

47. Lista S, Toschi N, Baldacci F, et al. Diagnostic accuracy of CSF neurofilament light chain protein in the biomarker-guided classification system for Alzheimer's disease. Neurochem. Int. 2017;108:355-360.

48. Meeter LHH, Dopper EG, Jiskoot LC, et al. Neurofilament light chain: a biomarker for genetic frontotemporal dementia. Ann. Clin. Transl. Neurol. 2016;3(8):623-636.

49. Mattsson N, Cullen NC, Andreasson U, et al. Association between longitudinal plasma neurofilament light and neurodegeneration in patients with Alzheimer disease. JAMA Neurol. 2019;76(7):791-799.

50. Neltner JH, Abner EL, Jicha GA, et al. Brain pathologies in extreme old age [Internet]. Neurobiol. Aging 2016;37:1-11.Available from: http://dx.doi.org/10.1016/j.neurobiolaging.2015.10.009

51. Jack Jr CR, Wiste HJ, Weigand SD, et al. Different definitions of neurodegeneration produce similar amyloid/neurodegeneration biomarker group findings. Brain 2015;138(12):3747-3759. 
52. Dickerson BC, Wolk D. Biomarker-based prediction of progression in MCI: comparison of AD-signature and hippocampal volume with spinal fluid amyloid- $\beta$ and tau. Front. Aging Neurosci. 2013;5:55.

53. McMillan CT, Avants BB, Cook P, et al. The power of neuroimaging biomarkers for screening frontotemporal dementia. Hum. Brain Mapp. 2014;35(9):4827-4840.

54. Ossenkoppele R, Mattsson N, Teunissen CE, et al. Cerebrospinal fluid biomarkers and cerebral atrophy in distinct clinical variants of probable Alzheimer's disease. Neurobiol. Aging 2015;36(8):2340-2347.

55. Bocti C, Rockel C, Roy P, et al. Topographical patterns of lobar atrophy in frontotemporal dementia and Alzheimer's disease. Dement. Geriatr. Cogn. Disord. 2006;21(5-6):364-372. 